\title{
Microbiology and Outcomes of Institutionalized Patients with Stroke-associated Pneumonia: A Prospective Observational Study
}

Jie Zhao

Zhejiang University School of Medicine

Lei-qing Li

Zhejiang University School of Medicine

Ning-xin Zhen

Zhejiang University School of Medicine

Lin-lin Du

Zhejiang University School of Medicine

Hui Shan

Zhejiang University School of Medicine

Yang Yu

Zhejiang University School of Medicine

Zhao-cai Zhang

Zhejiang University School of Medicine

Wei Cui

Zhejiang University School of Medicine

Bao-ping Tian ( $\boldsymbol{\nabla}$ TianBP@zju.edu.cn )

The Second Affiliated Hospital, Zhejiang University School of Medicine

Research

Keywords: stroke, pneumonia, microbiology, outcomes

Posted Date: March 25th, 2021

DOI: https://doi.org/10.21203/rs.3.rs-347961/v1

License: (c) (1) This work is licensed under a Creative Commons Attribution 4.0 International License. Read Full License 


\section{Abstract}

Background: The attributable mortality and microbial etiology of stroke-associated pneumonia (SAP) vary among different studies. We intednd to determine the microbiology and outcomes of SAP in lower respiratory tract (LRT) for patients with invasive mechanical ventilation (MV).

Methods: In this prospective observational study, included patients were divided into SAP and non-SAP based on comprehensive analysis of symptom, imaging and laboratory results. Baseline characteristic, clinical characteristic, microbiology and outcomes were recorded and evaluated.

Results: Of 185 patients, $41.6 \%$ developed SAP after onset of stroke, and they had lower proportion of non-smoker $(p=0.016)$, lower GCS score $(p<0.001)$, higher serum CRP score $(p<0.001)$ at ICU admission, and higher proportion of males $(p<0.001)$ and hypertension $(p=0.018)$ than patients with non-SAP. Gramnegative aerobic bacilli were the predominant organisms isolated (78\%), followed by Gram-positive aerobic cocci $(29.9 \%)$. Out of 19 isolated pathogens, main pathogens included K. pneumoniae, S. aureus, H. influenzae, A. baumannii, P. aeruginosa, E. aerogens, Serratia marcescens, and Burkholderia cepacia. SAP significantly prolonged length of MV $(p<0.001)$ and duration of ICU stays $(p<0.001)$, shorten MV-free days by $28(p<0.001)$, caused elevated vasopressor application $(p=0.002)$ and 60-day mortality $(p=0.001)$.

Conclusion: Microbiology of SAP is similar to early-phase HAP and VAP. SAP significantly prolongs duration of MV and lengths of ICU stays, but also markedly increase 60-day mortality.

Trial registration: ChiCTR2000028849; http://www.chictr.org.cn/index.aspx, Registered 05 January 2020.

\section{Background}

Pneumonia occurring within 7 days to onset of stroke was defined as SAP, and it was associated with prolonged hospital stays and poor prognosis[1-3]. Morbidity and attributable mortality of SAP vary among different studies, and the pathophysiology of SAP are mainly explained by aspiration and strokeinduced immunodepression[1]. Risk factors included dysphagia, impaired consciousness and ineffective cough reflex, as they impaired LRT ability of eliminating microbes from oropharyngeal contents and allow pathogens enter the lung $[4,5]$. Diverse communities of pulmonary microbiota have been discovered, thus dysbiosis was considered related to impaired pulmonary defenses $[4,6]$. Besides, the alternation of oral microbial diversity was not only observed on murine pneumonia model, but also among the patients' population of post-stroke and ventilation-associated pneumonia (VAP), inspiring us new directions investigating pathogenesis[4, 7-10]. In recent studies, elevated bronchoalveolar lavage (BAL) amylase level was associated with high risk of aspiration and positive-culture, while serum procalcitonin (PCT) was associated with prognosis of aspiration pneumonia (AP) [4, 5, 11-13]. However, diagnosis of SAP still relies on clinical features, as lack of gold standard biomarkers. According to current epidemiology of SAP, Staphylococcus aureus (S. aureus), Klebsiella pneumoniae (K. pneumoniae), Pseudomonas aeruginosa (P. aeruginosa), Escherichia coli (E. coli), Streptococcus pneumoniae (S. pneumoniae) and Haemophilus 
influenzae (H. influenzae) are considered as major pathogens, which is of high similarity with microbiology of hospital acquired pneumonia (HAP) or community acquired pneumonia (CAP) [1, 2, 1416]. Antibiotic prophylaxis has different effects in decreasing morbidity and mortality owing to distinctive subtypes of pneumonia as well as patient population $[6,17,18]$. Whether apply antibiotic prophylaxis or not and the choice of antibiotics remain controversial, and MV is also a risk factor of SAP, thus investigating microbiology of SAP of critically ill patients with MV is of high significance. We conduct this observative study in order to identify microbiology in the LRT and clinical features on such a strictly stratified population, to further optimize the converge of antibiotics and find significant medical indicators.

\section{Methods}

\section{Design and Population}

We conducted a prospective single-center observational study in an intensive care unit (ICU) of a Zhejiang University affiliated hospital between January 2020 and December 2020. The study complied with the current version of the Helsinki Declaration and appropriate clinical practice guidelines. The trial was approved by ethics committee of our hospital (2019 Ethical Review No.343), and registered in Chinese Clinical Trial Rsgistry (ChiCTR2000028849). Written informed consent was obtained from all of the included patients or the next of kin. Criteria for enrollment included ICU inpatient $\geq 18$, stroke onset within 72 hours, invasive MV at least for 24 hours. Acute ischemic stroke was defined as acute onset, focal neurological deficits or panfacial nerve dysfunction, presence of responsible lesion on imaging or duration of symptoms or signs for at least 24 hours, exclusion of non-vascular causes and cerebral hemorrhage. Acute hemorrhagic stroke was defined as acute onset, focal neurological deficit symptoms often accompanied by headache, vomiting, elevated blood pressure and varying degrees of disturbance of consciousness, head imaging showed bleeding lesions, exclusion of non-vascular cerebral etiology. SAP was defined as new or progressive pulmonary infiltrative lesions after stroke with at least two clinical symptoms, body temperature $\geq 38^{\circ} \mathrm{C}$, new cough, expectoration, purulent discharge, with or without chest pain, signs of lung consolidation, and/ or wet rales, leukocytosis $\left(>10,000 / \mathrm{mm}^{3}\right)$ or leukopenia $(<$ $4,000 / \mathrm{mm}^{3}$ ) with or without nuclear shift to the left. Exclusion criteria were infectious diseases (including pneumonia) within 3 months before the onset of stroke, antibiotics use within 3 months, comorbidities such as chronic obstructive pulmonary disease (COPD), interstitial lung disease, lung tumor, atelectasis, pulmonary edema, pulmonary embolism and autoimmunity diseases, medical history of dysphagia or gastroesophageal reflux, pregnant, pulmonary imaging did not accord with the manifestations of pneumonia after discussion by the research team, unclear medical history. Participants can withdraw from the study when they or reject to continue to take part in the study without any specific reason.

\section{Procedures and Statistical Analysis}

All enrolled patients were followed prospectively and managed according to the following protocol. Characteristic such as age, sex, type of stroke, previous medical history, time from onset of stroke to 
intubation as well as smoking status, and baseline data at ICU admission including Glasgow Coma Scale (GCS) score, white cell count (WBC), PCT, CRP were collected and recorded. Besides, outcomes such as duration of MV, duration of MV-free days by 28, duration of ICU stay, duration of hospital stay, vasopressor during ICU stay 60-day mortality were also observed and recorded. Presence of chest imaging examination indicted pulmonary inflammation when ICU admission, specimen should be obtained within 2 hours to ICU admission. Otherwise, continuous surveillance based on clinical symptoms were need, and chest imaging examination would be applied to these patients if symptoms appeared within 48 hours. Once positive imaging evidence supported inflammation, sample should be obtained as soon as possible. Quantitative analysis of fiberscope or semi-quantitative analysis of suction in endotracheal tube are used to collect specimens. The process of obtaining specimen, storage in sterile container and sending to laboratory should be finished within 2 hours (at room temperature). All specimens should be processed as soon as possible to ensure the activity of pathogenic bacteria, and strictly follow standard inspection procedures such as inoculation, culture, smear, staining and observation. The sputum samples were also inoculated in fungal culture medium. The automatic bacterial identification system (Merieux VITEK 2 Compact) was used for strain identification. After sample collection, empirical antibiotic therapy was started according to local epidemiology and antibiotic regimen would be modified if needed based on the bacterial culture results. Patients were monitored daily until discharge from the hospital or death after the diagnosis of pneumonia. Included patients were divided into SAP and non-SAP based on comprehensive analysis of symptom, imaging and laboratory results. Baseline charateritics and outcomes were analyzed and described as mean $\pm S D$, median (Interquartile range, IQR), percentage as appropriate. Contiunous variables were compared by Student's $\mathrm{t}$ test if they were normally distributed, otherwise, were compared by Wilcoxon Mann-Whitney U test. Pearson c2 test or Fisher exact test were applied to categorical variables to compare differences between SAP and non-SAP. All tests were 2-tailed and statistical significance was determined at an a level of 0.05. Statistical analyses were performed with the SPSS 20.0.

\section{Results}

\section{Comparison of Patients With SAP and With Non-SAP}

A total 392 patients with acute stroke were admitted at ICU during the study, 207 were excluded according to inclusion and exclusion criteria. Finally, 185 patients were eligible for further analysis and those were divided into two groups as SAP $(n=77)$ and non-SAP $(n=108)$ according to the microbiological results of sputum samples (Fig. 1). No participants were withdrawn from the trial, and all included patients completed the study protocol and assessment of the main outcomes. Comparing to patients with nonSAP, patients with SAP had lower GCS score (median: 8 vs 13, IQR: 4.25-10 vs $8-15, \mathbf{p}<0.001$ ), higher serum CRP score (median:10.6 vs 5.2, IQR:4.6-30.5 vs 1.8-13.4, p < 0.001) at ICU admission, higher proportion of males $(62 \%$ vs $36.1 \%, \mathbf{p}<0.001)$ and higher proportion of hypertension $(58.4 \%$ vs $40.7 \%, p=$ 0.018). Besides, comparing to non-SAP group, more patients in SAP group had smoking history ( $45.5 \%$ vs $25.9 \%, P=0.016$ ) (Table 1 ). No other baseline characteristics differed significantly between two groups. 
For both two groups, hemorrhagic stroke was the main type of stroke (93.5\%) and over fifty percent of patients had symptom of vomiting $(54.5 \%, 50.9 \%$, respectively, $\mathrm{p}=0.627)$ (Table 1$)$. 
Table 1

Baseline characteristics of the patients with and without microbiologically confirmed SAP at emergency admission

\begin{tabular}{|c|c|c|c|}
\hline Characteristics & $\begin{array}{l}\text { SAP } \\
(n=77)\end{array}$ & $\begin{array}{l}\text { Non-SAP } \\
(n=108)\end{array}$ & $\mathbf{P}$ \\
\hline Age, mean $\pm S D$ (year) & $56.0 \pm 15.2$ & $58.1 \pm 13.3$ & 0.325 \\
\hline Male, n (\%) & $48(62)$ & $39(36)$ & $<0.001$ \\
\hline Type of stroke & & & 0.997 \\
\hline Ischemic stroke, n (\%) & $5(6.5)$ & $7(6.5)$ & \\
\hline Hemorrhagic stroke, n (\%) & $72(93.5)$ & $101(93.5)$ & \\
\hline Vomiting, n (\%) & $42(54.5)$ & $55(50.9)$ & 0.627 \\
\hline $\begin{array}{l}\text { Time from onset of stroke to intubation, } \\
\text { median (IQR) (hr) }\end{array}$ & $23(10.5-29.8)$ & $24(12.3-36.0)$ & 0.287 \\
\hline \multicolumn{4}{|l|}{ At ICU admission } \\
\hline GCS, median (IQR) & $8(4.25-10)$ & $13(8-15)$ & $<0.001$ \\
\hline White blood cell count, mean $\pm \mathrm{SD}\left(\mathrm{X} 10^{9} / \mathrm{L}\right)$ & $12.1 \pm 5.0$ & $11.1 \pm 3.8$ & 0.112 \\
\hline C-reactive protein, median (IQR) (mg/L) & $10.6(4.6-30.5)$ & $5.2(1.8-13.4)$ & $<0.001$ \\
\hline Procalcitonin, median (IQR) (ng/mL) & $0.125(0.078-0.343)$ & $0.11(0.07-0.20)$ & 0.183 \\
\hline \multicolumn{4}{|l|}{ Comorbidities, n (\%) } \\
\hline Hypertension & $45(58.4)$ & $44(40.7)$ & 0.018 \\
\hline Heart disease & $5(6.5)$ & $10(9.3)$ & 0.497 \\
\hline Diabetes mellitus & $5(6.5)$ & $7(6.5)$ & 0.997 \\
\hline Renal disease & $4(5.2)$ & $3(2.8)$ & 0.647 \\
\hline Liver disease & $1(1.3)$ & $2(1.9)$ & 1.000 \\
\hline Smoking status & & & 0.016 \\
\hline Current smoker, n (\%) & $23(29.9)$ & $21(19.4)$ & \\
\hline Ex-smoker, n (\%) & $12(15.6)$ & $7(6.5)$ & \\
\hline Nonsmoker, n (\%) & $42(54.5)$ & $80(74.1)$ & \\
\hline
\end{tabular}




\section{Clinical Characteristics of Patients With Microbiologically Confirmed SAP}

Of these 77 patients, $48 \%$ had abnormal body temperature (median: 38.4 , IQR: $38.2-38.8,{ }^{\circ} \mathrm{C}$ ), and $67.5 \%$ patients had leukocytosis or leukopenia (median: 12.0, IQR: 8.75-13.95, X10 1 /L). Patients with purulent tracheobronchial aspirate occupied a higher proportion than those with non-purulent tracheobronchial aspirate (83\% vs $17 \%)$. According to chest radiograph, bilateral distribution (98.7\%) and pleural effusion (85.7\%) were important clinical characteristic, which appeared in almost all patients. Besides, serum CRP (median: 110, IQR: 57.45-166.55, mg/L) and serum PCT (median: 0.27, IQR: 0.135-0.713, ng/mL) were found elevated during ICU stay for patients with SAP (Table 2).

Table 2

Clinical characteristics of the patients with microbiologically confirmed SAP

\begin{tabular}{|ll|}
\hline Characteristics & SAP $(\mathbf{n}=77)$ \\
\hline Temperature $\leq 35.5^{\circ} \mathrm{C}$ or $\geq 38.5^{\circ} \mathrm{C}$ & \\
\hline $\mathrm{n}(\%)$ & $37(48)$ \\
\hline Temperature, median $(\mathrm{IQR})\left({ }^{\circ} \mathrm{C}\right)$, & $38.4(38.2-38.8)$ \\
\hline Leukocytosis $\left(\mathrm{WBC}>10 \times 10^{9} / \mathrm{L}\right)$ & \\
\hline or Leukopenia $\left(\mathrm{WBC}<4 \times 10^{9} / \mathrm{L}\right)$ & \\
\hline $\mathrm{N}(\%)$ & $52(67.5)$ \\
\hline WBC, median $(\mathrm{IQR})\left(\mathrm{X} 10^{9} / \mathrm{L}\right)$ & $12.0(8.75-13.95)$ \\
\hline Tracheobronchial aspirate, $\mathrm{n}(\%)$ & $64(83)$ \\
\hline Purulent & $13(17)$ \\
\hline Non-purulent & \\
\hline$\geq 2$ criteria & \\
\hline Chest radiograph, $\mathrm{n}(\%)$ & $66(85.7)$ \\
\hline Pleural effusion & $76(98.7)$ \\
\hline Bilateral & $25(32.5)$ \\
\hline Multilobar & $110(57.45-166.55)$ \\
\hline C-reactive protein, median $(\mathrm{IQR})(\mathrm{mg} / \mathrm{L})$ & $0.27(0.135-0.713)$ \\
\hline Procalcitonin, median $(\mathrm{IQR})(\mathrm{ng} / \mathrm{mL})$ & \\
\hline IQR = interquartile range & \\
\hline
\end{tabular}




\section{Microbial Etiology of Microbiologically Confirmed SAP}

Overall, 19 pathogens were isolated from deep sputum samplings for 77 patients in SAP group. 61 patients had one pathogen, for whom 44 was infected by Gram-negative aerobic bacilli, whereas 17 was infected by Gram-positive aerobic cocci. The remaining 16 patients were mixed infection, of which 14 patients were double infections and 2 patients were triple infections. Gram-negative aerobic bacilli were the predominant organisms isolated (78\%), followed by Gram-positive aerobic cocci (29.9\%). The majority of Gram-negative aerobic bacilli was K. pneumoniae (39\%). Other Gram-negative aerobic bacilli included Acinetobacter baumannii (A. baumannii), Enterobacter aerogenes (E. aerogens), Serratia marcescens, H. influenzae, Burkholderia cepacian, P. aeruginosa, Enterobacter asburiae (E. asburiae), Klebsiella aerogenes and Enterobacter kobei (E. kobei). As for Gram-positive aerobic cocci, S. aureus predominated (26\%), followed by S. pneumoniae and Methicillin-resistant S. aureus (MRSA) (Table 3). 
Table 3

Microbial etiology of SAP in LRT

\section{Microbiology}

Gram-negative aerobic bacilli

K. pneumoniae

A. baumannii

E. aerogens

Serratia marcescens

H. influenzae

Burkholderia cepacia

$P$. aeruginosa

E. asburiae

Klebsiella aerogenes

E. kobei

\section{Gram-positive aerobic cocci}

S.aureus

S. pneumoniae

MRSA

Multi-pathogen co-infection

Double infection

K. pneumoniae+ S. aureus

K. pneumoniae $+H$. influenzae

K. pneumoniae $+P$. aeruginosa

K. pneumoniae+ M.morganii

K. pneumonia + E. coli

S. aureus+ E. cloacae
$\operatorname{SAP}(n=77), n(\%)$

44 (57.1)

22 (28.6)

4 (5.2)

4 (5.2)

4 (5.2)

3 (3.9)

3 (3.9)

1 (1.3)

1 (1.3)

1 (1.3)

1 (1.3)

17 (22.1)

14 (18.2)

2 (2.6)

1 (1.3)

16 (20.8)

14 (18.2)

2 (2.6)

2 (2.6)

1 (1.3)

1 (1.3)

1 (1.3)

2 (2.6)

K. pneumoniae $=$ Klebsiella pneumoniae; A. baumannii $=$ Acinetobacter baumannii; E. aerogens $=$ Enterobacter aerogenes; $H$. influenzae $=$ Haemophilus influenzae; $P$. aeruginosa $=P$ seudomonas aeruginosa; $E$. asburiae = Enterobacter asburiae; $E$. kobei = Enterobacter kobei; S. aureus = Staphylococcus aureus; $S$. pneumoniae = Streptococcus pneumoniae; MRSA = Methicillin-resistant $S$. aureus; M. morganii = Morganella morganii; E. coli $=$ Escherichia coli; E. cloacae = Enterobacter cloacae; 
Microbiology

S. aureus $+H$. influenzae

S. aureus $+A$. baumannii

H. influenzae+ Branhamella catarrhalis

H. influenzae+ E. coli

P. aeruginosa + Burkholderia cepacia

Triple infection

K. pneumoniae+ P. aeruginosa + Carbapenem-resistant A. baumannii

$P$. aeruginosa $+A$. baumannii + Proteus mirabilis
$\operatorname{SAP}(n=77), n(\%)$

$1(1.3)$

$1(1.3)$

$1(1.3)$

$1(1.3)$

$1(1.3)$

$2(2.6)$

$1(1.3)$

$1(1.3)$

K. pneumoniae $=$ Klebsiella pneumoniae; $A$. baumannii $=$ Acinetobacter baumannii; E. aerogens $=$ Enterobacter aerogenes; $H$. influenzae = Haemophilus influenzae; $P$. aeruginosa $=$ Pseudomonas aeruginosa; E. asburiae = Enterobacter asburiae; E. kobei $=$ Enterobacter kobei; S. aureus = Staphylococcus aureus; S. pneumoniae = Streptococcus pneumoniae; MRSA = Methicillin-resistant S. aureus; M. morganii = Morganella morganii; E. coli = Escherichia coli; E. cloacae = Enterobacter cloacae;

\section{Antimicrobial Therapy of Post-stroke Patients With SAP or Non-SAP}

In terms of antibiotic use in patients with SAP, post-operative application predominated, followed by preoperative and post-operative antibiotic coverage. As for antimicrobial therapy in patients with non-SAP, post-operative application predominated, followed by without antibiotic coverage, then pre-operative and post-operative antibiotic coverage (Table S1). The difference of the medication regimen of antibiotics between the two groups was statistically significant $(p<0.001)$. Antibiotics use included cefuroxime, piperacillin-tazobactam, clindamycin, cefotaxime, cefoperazone-sulbactam, latamoxef and ceftriaxone. Besides, microbiology of patients with SAP and the corresponding antibiotics regimen were illustrated in the Table S2.

\section{Outcome Comparison of Patients With SAP and Non-SAP}

Compared to patients with SAP (median: 148, IQR: 92.5-238, hr), those with non-SAP spent less time on MV (median: 29.5, IQR: 14-133.25, p < 0.001). Consistently, patients with non-SAP (median: 27, IQR: 22.527, days) have more MV-free days by 28 than patients with SAP (median: 22, IQR: 18-24, p < 0.001). SAP significantly prolonged duration of ICU stays compared with non-SAP (median: 9 vs 3, IQR: $5-14$ vs 27.75 , days, $p<0.001)$, but didn't affect the length of hospitalization $(p=0.079)$. More patients with SAP ( $n$ $=21,27.3 \%)$ were applied vasopressor during ICU stays than patients with non-SAP $(n=11,10.2 \%, p=$ 0.002). In addition, significant difference in 60-day mortality was also found between patients with SAP $(n=17,22 \%)$ and patients with non-SAP $(n=6,5.6 \%, p=0.001)$ (Table 4$)$. 
Table 4

Outcomes of the patients with microbiologically confirmed SAP compared with matched control

\begin{tabular}{|llll|}
\hline Characteristics & SAP & Non-SAP & P \\
\hline Duration of mechanical ventilation, median (IQR) (hr) & $\begin{array}{l}148(92.5- \\
(\mathbf{n}=108)\end{array}$ & $\begin{array}{l}29.5(14- \\
133.25)\end{array}$ & 0.001 \\
\hline $\begin{array}{l}\text { Mechanical ventilation-free days by 28, median (IQR) } \\
\text { (day) }\end{array}$ & $22(18-24)$ & $27(22.5-27)$ & 0.001 \\
\hline Duration of ICU stay, median (IQR) (day) & $9(5-14)$ & $3(2-7.75)$ & $<$ \\
\hline Duration of hospital stay, median (IQR) (day) & $13(9-18.5)$ & $11(8.25-16)$ & 0.079 \\
\hline Vasopressor during ICU stay, n\% & $21(27.3)$ & $11(10.2)$ & 0.002 \\
\hline 60-day mortality, n\% & $17(22)$ & $6(5.6)$ & 0.001 \\
\hline IQR = interquartile range, hr = hour & & & \\
\hline
\end{tabular}

\section{Discussion}

This was the first survey investigating microbiology of SAP in LRT for post-stroke patients treated with invasive MV. In our study, the main attributed pathogens were K. pneumoniae (39\%), S. aureus (26\%), H. influenzae (10.4\%), A. baumannii (7.8\%), P. aeruginosa (6.5\%), E. aerogens (5.2\%), Serratia marcescens (5.2\%), Burkholderia cepacia (5.2\%), similar to the microbiology of culture-positive pneumonia for hospitalized neurologic patients and early-onset VAP[19-21]. Besides, a systemic review involving 7968 patients with acute stroke indicted that the commonly isolated organisms of SAP included $K$. pneumoniae (12.8\%), E. coli (9\%), S. aureus (10.1\%), P. aeruginosa (6\%), A. baumanii (4.6\%) and S. pneumoniae (3.5\%)[22]. As for the type of pathogens, our conclusion was of essential agreement with the systemic review, however, the specific frequency for each pathogen calculated by different studies varied. This difference might be explained by many divergences among studies including heterogeneity of patients' population, heterogeneity of epidemics in different region, heterogeneity of clinical environment, whether treated with invasive treatment or prophylactic antibiotic therapy (PAT) and so on. For instance, this systemic analysis pointed out that studies whose patients were at relatively higher-risk such as dysphagic or ICU admission had a high proportion of Gram-negative aerobic bacilli and S. aureus, which is consistent with findings in our study[22].

Critically ill patients deserve more attention, as they have many risk factors of SAP, including hypertension, dysphagia, MV, male, a lower consciousness level and bedridden[23]. In our study, hemorrhagic stroke was the main type of stroke. Actually, ischemic stroke is the more common type of stroke, and most researchers always recruited patients from stroke units (ischemic stroke predominated) or directly selected patients with ischemic stroke[22, 24, 25]. The difference between ischemic stroke and 
hemorrhagic stroke was lying on many aspects including mechanism, symptom as well as prognosis, whereas whether these divergences would further impact SAP are still unclear. For example, strokeinduced immunodepression was found correlated with mechanism and morbidity risk of SAP for patients with ischemic stroke[26, 27]. However, whether immunodepression induced by hemorrhagic stroke is identical to ischemic stroke still uncertain. In our prospective cohort study, serum CRP and PCT, increased vigorously comparing with the baseline level at ICU admission. Serum CRP and PCT were considered to be independently associated with SAP in some studies, but not others. The diagnostic value of serum CRP and PCT in discrimination of SAP was not definite yet[28]. Improvement of diagnostic sensitivity and specificity need comprehensive consideration of symptoms and biomarkers. For afebrile patients with chest findings and leucocytosis or new radiological chest infiltrates, Kalra et al highly recommended CRP $\geq 30 \mathrm{mg} / \mathrm{L}$ as a supplement to diagnosed SAP[29]. This strategy remedied missed diagnosis owing to afebrile to some extent. In our study, CRP at ICU admission elevated mildly in SAP group (10.6 vs 5.2 $\mathrm{mg} / \mathrm{L}, \mathrm{p}<0.001)$ than non-SAP, which might be a somatic response to acute stroke or correlate with stroke-induced immunodepression. However, whether such CRP change in early phase was a valuable sign helping recognize potential patient with SAP, are still unknown. In addition, dynamic change process of biomarkers (eg. CRP and PCT) reflect the progression and prognosis of disease to some extent. PCT were considered to have higher diagnostic and prognostic accuracy of SAP than CRP in recent studies[30, 31]. Interestingly, PCT-guided antimicrobial strategies in ICU attracted a lot attention, and PCT-guided cessation of antibiotics were found to reduce both antibiotic exposure and mortality[32, 33]. PCT might be a good indicator reflecting progression of SAP and guiding clinical medication. More studies should be designed to clarify the details about role of the serum CRP and PCT in SAP. We found patients with SAP spent more time on MV and staying in ICU, were applied more vasopressors, and had higher 60-day mortality than patients with non-SAP. This conclusion is consistent with the findings in previous studies, in which SAP was associated with poor functional outcome and mortality[34, 35]. It is really important to figure out how to recognize potential patients as early as possible and how to take active strategies to avoid the occurrence of SAP.

Some patients were applied antibiotics before operation because they indeed faced high-risk of infection and had a poor physical status in our study. Although antibiotics use was discouraged to prevent SAP according to current guidance, physicians still would choose PAT in practical clinical work, considering the infectious threat patients faced and low probability of victory of anti-infection only relying on themselves. Whether PAT favors preventing infection and prognosis is a controversial topic, and several studies have been performed[18, 36-38]. Two multicenter, open-label, randomized controlled trials with masked endpoint assessment have been published. The Preventive Antibiotics in Stroke Study (PASS) conducted by Westendorp et al explored the positive effects of ceftriaxone on outcomes for patients with acute stroke, whereas Lalit et al investigated whether prophylactic antibiotics could reduce pneumonia in post-stroke patients with dysphagia (STROKE-INF) $[18,37]$. The former found PAT could reduce incidence of infection, while the latter illustrated that PAT did not reduce the frequency of pneumonia. The consistent conclusion in these two high-quality studies was that PAT did not improve functional outcome at 3 months. The above findings suggested PAT was only capable of preventing urinary tract infections, 
not SAP[39]. In summary, in terms of bactericidal effect of antibiotics, no antibiotics have been found to have beneficial effects in preventing SAP so far. However, specific antibiotic protocols in each study succumb to local hygiene policies based on local epidemiology, and influenced by local empiric administration habits. Several factors such as type of antibiotics, time point of administration, dose, mode of administration, type of population will impact the final effects of antibiotics. We haven't found the positive effects of PAT in SAP so far, probably because we didn't choose the optimal antibiotics strategies in previous studies. Another point attracted our attention is the neuroprotective effects of some antibiotics. Previously, ceftriaxone was found to significantly reduce acute stroke mortality in animal models, and these positive results might be due to its neuroprotective effects including increasing glutamate uptake and inducing neurotrophins[40]. Recently, Dai et al found that minocycline could attenuate brain injury and iron overload after intracerebral hemorrhage in aged female rats[41]. Besides, Yang et al also demonstrated that minocycline could attenuate acute stroke injury in rat brain by promoting blood-brain barrier remodeling and facilitating neuroprotective phenotype alternative activation of microglia / macrophages. Treatment with minocycline significantly reduced levels of proinflammatory factors tumor necrosis factors- $\alpha$ (TNF- $\alpha$ ) and interleukin-1 $\beta$ (IL-1 $\beta)$ and increased levels of anti-inflammatory factors transforming growth factor- $\beta$ (TGF- $\beta$ ) and interleukin-10 (IL-10)[42]. As we all known, neurovascular injury is the primary etiology of SAP, antibiotics would have application value in early phase of stoke if they have neuroprotective effects. Thus, this direction needs more attention and more investigations should be conducted to clarify more details. In summary, it is insufficient to totally negate the effects of PAT in SAP just depending on evidence from current studies.

There are some limitations in our study. Firstly, our study is single-center observational cohort study and the sample size was relatively small. Since microbiology of SAP associate with local epidemiology, our findings need to be further validated in a larger cohort based on multi-center cooperation. Secondly, our study only focused on patients' short-term outcomes such as duration of ICU stays and 60-day mortality. Long-term follow-up should continue to figure out the effects of SAP in long-term outcome. Thirdly, as microbiologically confirmed bacterial pneumonia was the focus in our study, determination of infection strictly followed results of sputum bacterial culture, whereas virus detection was not performed in our study. It would be better when broader pathogen (eg. bacterium and viruses) distribution and frequency were figured out. Finally, some patients were applied pre-operative prophylactic antibiotics since they had high-risk of infection and serious status, which might impact the results of bacterial culture. The main antibiotics in our study was cefuroxime, which is a broad-spectrum antibiotic. Thus, the actual morbidity of SAP might be higher than results obtained in our study.

\section{Conclusion}

Overall, this is the first prospective cohort study to investigate the microbiology of SAP in LRT for critically post-stroke patients treated with invasive MV. Those patients are at high risk of developing SAP, especially when patients have the following characteristic: male, have smoking history, hypertension, unconsciousness, elevated CRP at ICU admission etc. We found the main pathogens responsible for SAP in LRT were K. pneumoniae, S. aureus, H. influenzae, A. baumanni, P. aeruginosa, E. aerogens, Serratia 
marcescens, Burkholderia cepacia and etc, which is similar to microbiology of early-phase HAP and VAP. Besides, SAP would significantly not only prolong duration of MV and duration of ICU stay, but also increase vasopressor during ICU stays as well as mortality.

\section{Declarations}

\section{Ethics approval and consent to participate}

The trial was approved by ethics committee of our hospital (2019 Ethical Review No.343), and registered in Chinese Clinical Trial Rsgistry (ChiCTR2000028849). Written informed consent was obtained from all of the included patients.

\section{Consent for publication}

Not applicable

\section{Availability of data and materials}

The datasets used and/or analysed during the current study are available from the corresponding author on reasonable request.

\section{Competing interests}

The authors declare that they have no competing interests

\section{Funding}

This work was supported by the National Natural Science Foundation of China (81700023), the Zhejiang Provincial Natural Science Foundation of China (LY20H010002) and the Medical and Health Research Program of Zhejiang Province (2019RC181) to TBP.

\section{Authors' contributions:}

TBP and LLQ contributed the conception and design of this study. ZJ and ZNX acquired the data. DLL, SH and $\mathrm{CW}$ analyzed and interpreted the data. ZJ and TBP drafted the manuscript. ZJ, LLQ and YY conducted statistical analysis. TBP, ZZC and CW supervised the study. All authors reviewed drafts of the manuscript and approved the final version.

\section{Acknowledgements}

Not applicable

\section{Abbreviations}


Pneumonia in Stroke Consensus, PISCES; stroke-associated pneumonia, SAP; lower respiratory tract, LRT; bronchoalveolar lavage, BAL; C-reactive protein, CRP; aspiration pneumonia, AP; procalcitonin, PCT; ventilation-associated pneumonia, VAP; Staphylococcus aureus, S. aureus; Klebsiella pneumoniae, K. pneumoniae; Pseudomonas aeruginosa, P. aeruginosa; Escherichia coli, E. coli; Streptococcus pneumoniae, S. pneumoniae; Haemophilus influenzae, H. influenzae; hospital acquired pneumonia, HAP; community acquired pneumonia, CAP; mechanical ventilation, $\mathrm{MV}$; intensive care unit, ICU; chronic obstructive pulmonary disease, COPD; Glasgow Coma Scale, GCS; white cell count, WBC; Acinetobacter baumannii, A. baumannii; Enterobacter aerogenes, E. aerogens; Enterobacter asburiae, E. asburiae; Enterobacter kobei, E. kobei; Methicillin-resistant S. aureus, MRSA; prophylactic antibiotic treatment, PAT; Preventive Antibiotics in Stroke Study, PASS; necrosis factors-a, TNF- $\alpha$; interleukin-1 $\beta$, IL-1 $\beta$; transforming growth factor- $\beta$, TGF- $\beta$; interleukin-10, IL-10

\section{References}

1. Hannawi Y, Hannawi B, Rao CP, Suarez JI, Bershad EM. Stroke-associated pneumonia: major advances and obstacles. Cerebrovasc Dis. 2013;35(5):430-43.

2. Hassan A, Khealani BA, Shafqat S, Aslam M, Salahuddin N, Syed NA, Baig SM, Wasay M. Strokeassociated pneumonia: microbiological data and outcome. Singapore Med J. 2006;47(3):204-7.

3. Ferrer M, Torres A. Epidemiology of ICU-acquired pneumonia. Curr Opin Crit Care. 2018;24(5):325-31.

4. Mandell LA, Niederman MS. Aspiration Pneumonia. N Engl J Med. 2019;380(7):651-63.

5. DiBardino DM, Wunderink RG. Aspiration pneumonia: a review of modern trends. J Crit Care. 2015;30(1):40-8.

6. Wunderink RG, Waterer G. Advances in the causes and management of community acquired pneumonia in adults. BMJ. 2017;358:j2471.

7. Morinaga Y, Take Y, Sasaki D, Ota K, Kaku N, Uno N, Sakamoto K, Kosai K, Miyazaki T, Hasegawa H, et al. Exploring the microbiota of upper respiratory tract during the development of pneumonia in a mouse model. PLoS One. 2019;14(9):e0222589.

8. Emonet S, Lazarevic V, Leemann Refondini C, Gaïa N, Leo S, Girard M, Nocquet Boyer V, Wozniak H, Després $L$, Renzi G, et al. Identification of respiratory microbiota markers in ventilator-associated pneumonia. Intensive Care Med. 2019;45(8):1082-92.

9. Boaden E, Lyons M, Singhrao SK, Dickinson H, Leathley M, Lightbody CE, McLoughlin A, Khan Z, Crean S, Smith C, et al. Oral flora in acute stroke patients: A prospective exploratory observational study. Gerodontology. 2017;34(3):343-56.

10. Chiu CY, Miller SA. Clinical metagenomics. Nat Rev Genet. 2019;20(6):341-55.

11. Weiss CH, Moazed F, DiBardino D, Swaroop M, Wunderink RG. Bronchoalveolar lavage amylase is associated with risk factors for aspiration and predicts bacterial pneumonia. Crit Care Med. 2013;41(3):765-73. 
12. Legriel S, Grigoresco B, Martel P, Henry-Lagarrigue M, Lvovschi V, Troché G, Amara M, Jacq G, Bruneel F, Bernard M, et al. Diagnostic Accuracy of Procalcitonin for Early Aspiration Pneumonia in Critically III Patients with Coma: A Prospective Study. Neurocrit Care. 2019;30(2):440-8.

13. El-Solh AA, Vora H, Knight PR, Porhomayon J. Diagnostic use of serum procalcitonin levels in pulmonary aspiration syndromes. Crit Care Med. 2011;39(6):1251-6.

14. Chen LF, Chang CY, Hsu LC, Tsai PH, Chang SJ, Chang SC, Yuan MK, Lai YC, Liu YC, Wang WS. Bacterial pneumonia following acute ischemic stroke. J Chin Med Assoc. 2013;76(2):78-82.

15. Lin P, Fu JX, Zhang HN, Wang K. Analysis of pathogens, risk factors, and T lymphocyte levels in patients with pneuomonia following a stroke. Journal of Pathogen Biology. 2019;14(07):826-9.

16. Mingmei Z, Fan W, Lin Z, Song P, Jin Z, Mingwei H. Analysis of risk factors and pathogens for strokeassociated penumonia in intensive care unit. Chin J Emerg Med. 2015;24(09):1004-10.

17. Leone M, Bouadma L, Bouhemad B, Brissaud O, Dauger S, Gibot S, Hraiech S, Jung B, Kipnis E, Launey Y, et al. Hospital-acquired pneumonia in ICU. Anaesth Crit Care Pain Med. 2018;37(1):83-98.

18. Kalra L, Irshad S, Hodsoll J, Simpson M, Gulliford M, Smithard D, Patel A, Rebollo-Mesa I. Prophylactic antibiotics after acute stroke for reducing pneumonia in patients with dysphagia (STROKE-INF): a prospective, cluster-randomised, open-label, masked endpoint, controlled clinical trial. Lancet. 2015;386(10006):1835-44.

19. Lee HS, Moon J, Shin HR, Ahn SJ, Kim TJ, Jun JS, Lee ST, Jung KH, Park KI, Jung KY, et al. Pneumonia in hospitalized neurologic patients: trends in pathogen distribution and antibiotic susceptibility. Antimicrob Resist Infect Control. 2019;8:25.

20. Kalanuria AA, Zai W, Mirski M. Ventilator-associated pneumonia in the ICU. Crit Care. 2014;18(2):208.

21. Nair GB, Niederman MS. Ventilator-associated pneumonia: present understanding and ongoing debates. Intensive Care Med. 2015;41(1):34-48.

22. Kishore AK, Vail A, Jeans AR, Chamorro A, Di Napoli M, Kalra L, Langhorne P, Roffe C, Westendorp W, Nederkoorn PJ, et al. Microbiological Etiologies of Pneumonia Complicating Stroke: A Systematic Review. Stroke. 2018;49(7):1602-9.

23. Liu DD, Chu SF, Chen C, Yang PF, Chen NH, He X. Research progress in stroke-induced immunodepression syndrome (SIDS) and stroke-associated pneumonia (SAP). Neurochem Int. 2018.

24. Kalra L, Hodsoll J, Irshad S, Smithard D, Manawadu D. Comparison of the diagnostic utility of physician-diagnosed with algorithm-defined stroke-associated pneumonia. J Neurol Neurosurg Psychiatry. 2016;87(11):1163-8.

25. Warusevitane A, Karunatilake D, Sim J, Lally F, Roffe C. Safety and effect of metoclopramide to prevent pneumonia in patients with stroke fed via nasogastric tubes trial. Stroke. 2015;46(2):454-60.

26. Hoffmann S, Harms H, Ulm L, Nabavi DG, Mackert BM, Schmehl I, Jungehulsing GJ, Montaner J, Bustamante A, Hermans $M$, et al. Stroke-induced immunodepression and dysphagia independently predict stroke-associated pneumonia - The PREDICT study. J Cereb Blood Flow Metab. 2017;37(12):3671-82. 
27. Urra X, Laredo C, Zhao Y, Amaro S, Rudilosso S, Renú A, Prats-Galino A, Planas AM, Oleaga L, Chamorro Á. Neuroanatomical correlates of stroke-associated infection and stroke-induced immunodepression. Brain Behav Immun. 2017;60:142-50.

28. Smith CJ, Kishore AK, Vail A, Chamorro A, Garau J, Hopkins SJ, Di Napoli M, Kalra L, Langhorne P, Montaner J, et al. Diagnosis of Stroke-Associated Pneumonia: Recommendations From the Pneumonia in Stroke Consensus Group. Stroke. 2015.

29. Kalra L, Smith CJ, Hodsoll J, Vail A, Irshad S, Manawadu D. Elevated C-reactive protein increases diagnostic accuracy of algorithm-defined stroke-associated pneumonia in afebrile patients. Int J Stroke. 2019;14(2):167-73.

30. Wang C, Gao L, Zhang ZG, Li YQ, Yang YL, Chang T, Zheng LL, Zhang XY, Man MH, Li LH. Procalcitonin Is a Stronger Predictor of Long-Term Functional Outcome and Mortality than HighSensitivity C-Reactive Protein in Patients with Ischemic Stroke. Mol Neurobiol. 2016;53(3):1509-17.

31. Shi G, Li M, Zhou R, Wang X, Xu W, Yang F, Xue S. Procalcitonin related to stroke-associated pneumonia and clinical outcomes of acute ischemic stroke after IV rt-PA treatment. Cell Mol Neurobiol. 2021.

32. Lam SW, Bauer SR, Fowler R, Duggal A. Systematic Review and Meta-Analysis of ProcalcitoninGuidance Versus Usual Care for Antimicrobial Management in Critically III Patients: Focus on Subgroups Based on Antibiotic Initiation, Cessation, or Mixed Strategies. Crit Care Med. 2018;46(5):684-90.

33. Huang HB, Peng JM, Weng L, Wang CY, Jiang W, Du B. Procalcitonin-guided antibiotic therapy in intensive care unit patients: a systematic review and meta-analysis. Ann Intensiv Care. 2017;7(1):114.

34. Suda S, Aoki J, Shimoyama T, Suzuki K, Sakamoto Y, Katano T, Okubo S, Nito C, Nishiyama Y, Mishina $\mathrm{M}$, et al. Stroke-associated infection independently predicts 3-month poor functional outcome and mortality. Journal of neurology. 2018;265(2):370-5.

35. Finlayson O, Kapral M, Hall R, Asllani E, Selchen D, Saposnik G. Risk factors, inpatient care, and outcomes of pneumonia after ischemic stroke. Neurology. 2011;77(14):1338-45.

36. Smith CJ, Heal C, Vail A, Jeans AR, Westendorp WF, Nederkoorn PJ, van de Beek D, Kalra L, Montaner $\mathrm{J}$, Woodhead $\mathrm{M}$, et al. Antibiotic Class and Outcome in Post-stroke Infections: An Individual Participant Data Pooled Analysis of VISTA-Acute. Front Neurol. 2019;10:504.

37. Westendorp WF, Vermeij JD, Zock E, Hooijenga IJ, Kruyt ND, Bosboom HJ, Kwa VI, Weisfelt M, Remmers MJ, ten Houten R, et al. The Preventive Antibiotics in Stroke Study (PASS): a pragmatic randomised open-label masked endpoint clinical trial. Lancet. 2015;385(9977):1519-26.

38. Shim R, Wong CH. Ischemia. Immunosuppression and Infection-Tackling the Predicaments of PostStroke Complications. International journal of molecular sciences. 2016;17(1).

39. Meisel A, Smith CJ. Prevention of stroke-associated pneumonia: where next? Lancet. 2015;386(10006):1802-4. 
40. Thöne-Reineke C, Neumann C, Namsolleck P, Schmerbach K, Krikov M, Schefe JH, Lucht K, Hörtnagl $\mathrm{H}$, Godes $\mathrm{M}$, Müller $\mathrm{S}$, et al. The beta-lactam antibiotic, ceftriaxone, dramatically improves survival, increases glutamate uptake and induces neurotrophins in stroke. J Hypertens. 2008;26(12):2426-35.

41. Dai SH, Hua Y, Keep RF, Novakovic N, Fei Z, Xi GH. Minocycline attenuates brain injury and iron overload after intracerebral hemorrhage in aged female rats. Neurobiol Dis. 2019;126:76-84.

42. Yang Y, Salayandia VM, Thompson JF, Yang LY, Estrada EY, Yang Y. Attenuation of acute stroke injury in rat brain by minocycline promotes blood-brain barrier remodeling and alternative microglia/macrophage activation during recovery. J Neuroinflammation. 2015;12:26-6.

\section{Figures}

\section{2 patients with acute stroke at ICU admisssionwere eligible}

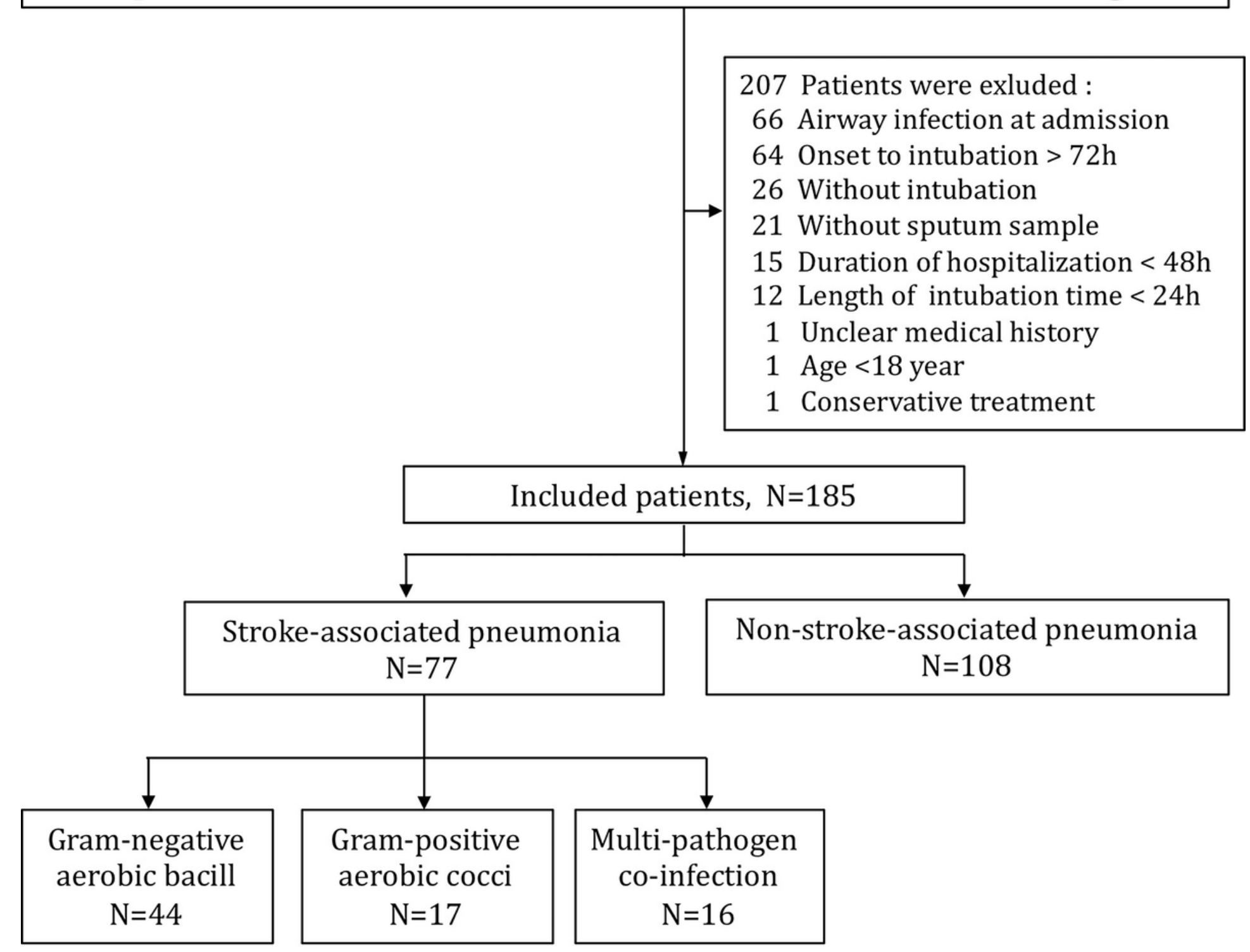

Figure 1 
Flow diagram of specific process of the trial, illustrating the number of patients in each step and each group.

\section{Supplementary Files}

This is a list of supplementary files associated with this preprint. Click to download.

- Additionalfile1.docx

- floatimage1.jpeg 